\title{
Retraso prehospitalario en pacientes con infarto agudo de miocardio en la Ciudad de México
}

\author{
Pre-Hospital delay of patients with ST-elevation myocardial infarction in Mexico City \\ Diego Araiza-Garaygordobil1*, Hector González-Pacheco, Carlos Sierra-Fernández, \\ Francisco Azar-Manzur ${ }^{1}$, José L. Briseño-De la Cruz ${ }^{1}$, Marco A. Martínez-Ríos², \\ Carlos Martínez-Sánchez ${ }^{1}$ y Alexandra Arias-Mendoza ${ }^{1}$ \\ ${ }^{1}$ Unidad Coronaria; ${ }^{2}$ Dirección. Instituto Nacional de Cardiología Ignacio Chávez, Ciudad de México, México
}

Sr. Editor,

En México, el $47.4 \%$ de los pacientes con infarto agudo de miocardio con elevación del segmento ST (IAMCEST) no reciben terapia de reperfusión ${ }^{1}$. Si bien múltiples factores contribuyen a este fenómeno², el retraso hasta la reperfusión (atribuible al paciente o al sistema de salud) podría constituir una barrera importante a la reperfusión oportuna. El objetivo de este trabajo es evaluar el tiempo de retraso prehospitalario (tiempo inicio-a-puerta, onset-to door) en pacientes mexicanos con IAMCEST.

Realizamos un estudio descriptivo y retrospectivo del tiempo inicio-a-puerta en pacientes mayores de 18 años con diagnóstico final de IAMCEST atendidos en un centro de atención cardiovascular de la Ciudad de México entre enero de 2013 y mayo de 2016. Se incluyeron pacientes reperfundidos (inicio de fibrinólisis 0 angioplastia antes de las primeras 12 horas) y no reperfundidos. Los pacientes del estudio se presentaron directamente o fueron referidos al centro de estudio para recibir fibrinólisis, angioplastia primaria o para completar estrategia farmacoinvasiva; este hospital es el único que ofrece servicio de angioplastia primaria 24/7 para pacientes no derechohabientes en la Ciudad de México.
Este trabajo se centró en el tiempo inicio-a-puerta, que contempla el intervalo desde el inicio de los síntomas hasta la llegada a puerta de un hospital con capacidad de angioplastia o fibrinólisis ${ }^{3}$. Simultáneamente se analizó el tiempo de inicio de síntomas a primer contacto médico (time-to-first medical contact). La información se obtuvo del expediente clínico electrónico: si los datos no estuvieron disponibles, el caso se excluyó del estudio. Para el análisis estadístico, las variables categóricas se describieron mediante porcentajes y las variables continuas con distribución no normal mediante medianas y rango intercuartílico (RIC).

Un total de 1,494 pacientes con IAMCEST fueron incluidos en la muestra. Los pacientes mayoritariamente fueron hombres $(83.7 \%)$, con hipertensión arterial sistémica $(47.0 \%)$, historia de uso de tabaco $(33.2 \%)$ y antecedentes de diabetes mellitus tipo 2 (38.8\%). La tasa de reperfusión fue del $62.7 \%$, de los cuales 451 (30.1\%) recibieron fibrinólisis y 487 (32.6\%) angioplastia primaria.

La información sobre el tiempo de retraso prehospitalario se encontró en el $91.4 \%(n=1,365)$ de los expedientes. La mediana de tiempo inicio-a-puerta fue 648 [RIC: 371748] min. La mediana de tiempo a primer contacto médico fue de 138 min [RIC: 87-152]. Entre los pacientes que no recibieron reperfusión (510; 37.3\%), el 26.8\% (137)

\section{Correspondencia:}

*Diego Araiza-Garaygordobil

Juan Badiano, 1

Col. Belisario Domínguez Sección XVI, Del. Tlalpan

C.P. 14080 , Ciudad de México, México

E-mail: dargaray@gmail.com

Fecha de recepción: 13-09-2017

Fecha de aceptación: 28-08-2018

DOI: 10.24875/ACM.M19000019
Disponible en internet: 08-05-2019 Arch Cardiol Mex. 2019;89(2):188-190 www.archivoscardiologia.com

1405-9940 @ 2018 Instituto Nacional de Cardiología Ignacio Chávez. Publicado por Permanyer México SA de CV. Este es un artículo Open Access bajo la licencia CC BY-NC-ND (http://creativecommons.org/licenses/by-nc-nd/4.0/). 


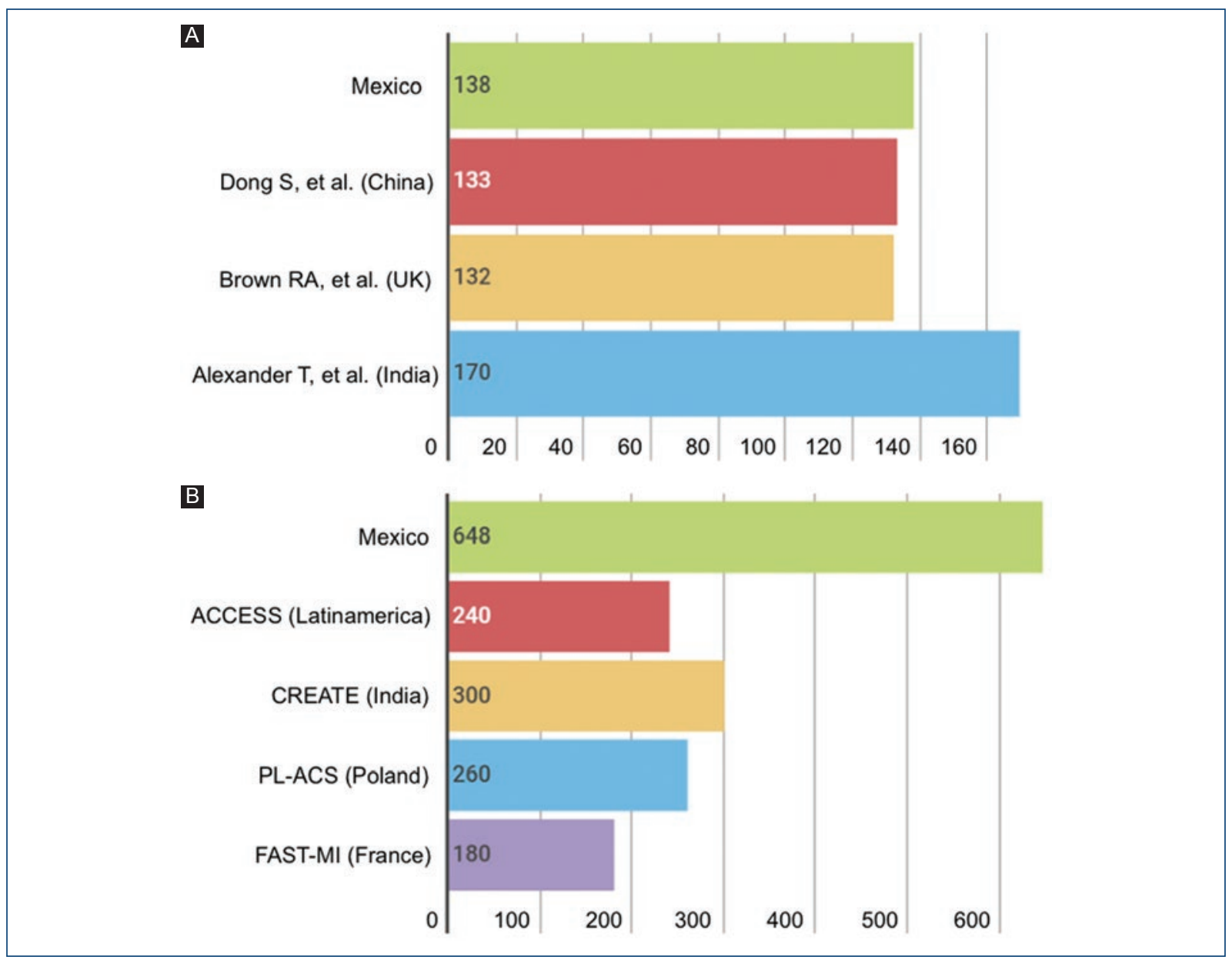

Figura 1. A: Comparación del tiempo al primer contacto médico en pacientes con infarto agudo de miocardio con elevación del segmento ST (IAMCEST) en México y otros países (mediana en minutos). B: Comparación del tiempo inicio-a-puerta en pacientes con IAMCEST en México y otros países (mediana en minutos).

ACCESS: ACute Coronary Events - a multinational Survey of current management Strategies; CREATE: Treatment and Outcomes of Acute Coronary Syndromes in India; FAST MI: French Registry on Acute ST-Elevation Myocardial Infarction; PL ACS: Polish Registry of Acute Coronary Syndromes.

tuvieron un primer contacto médico dentro de las primeras 12 horas desde el inicio de los síntomas.

El tiempo total de isquemia es un factor asociado a desenlaces como mortalidad, tamaño final del infarto e insuficiencia cardíaca ${ }^{4}$. Varios factores pueden influir en el tiempo total de isquemia, incluyendo variables geográficas, culturales, sociales y relacionadas al sistema de salud. El retraso prehospitalario (onset-to-door time) puede dividirse en dos: el retraso asociado al paciente (tiempo de primer contacto médico) y al sistema (primer contacto médico a puerta). El retraso del paciente depende de factores sociales y geográficos como la accesibilidad a servicios de salud, y factores culturales sobre la percepción y el significado de sus síntomas. Al comparar los resultados de nuestro estudio con otros registros, el tiempo a primer contacto médico en México es similar al observado en otros países (Fig. 1A). Registros de China, Reino Unido e India han reportado tiempos de primer contacto médico de 132-170 minutos ${ }^{5,6}$ en comparación con los 138 minutos observados en nuestro estudio.

Es el retraso del sistema en México el que resulta preocupante. En este trabajo se identificó una mediana de 510 minutos ( 8.5 horas), resultando en un tiempo inicio-a-puerta de 648 minutos. Registros de síndromes coronarios agudos en países como Polonia $(\mathrm{PL}-\mathrm{ACS})^{7}$ y Francia $(\mathrm{FAST}-\mathrm{MI})^{8}$ han reportado tiempos inicio-a-puerta de 180 a 260 minutos. En países en desarrollo, los registros ACCESS ${ }^{9}$ (Latinoamérica, África y Oriente Medio) y CREATE $^{10}$ (India) reportaron 240 a 300 minutos. Nuestro estudio muestra un tiempo inicio-a-puerta que duplica el reportado en el registro CREATE y por lo menos triplica los resultados del 
registro francés (Fig. 1B). Las causas detrás de este fenómeno pueden ser múltiples: la extensión geográfica del área metropolitana de la Ciudad de México, las condiciones de tráfico, la fragmentación del sistema de salud en diferentes proveedores, deficiencias en la capacidad diagnóstica y de referencia, etc.

En este estudio no se ahondó sobre el tiempo puerta-aguja o tiempo puerta-balón, subintervalos de tiempo que, si bien son ampliamente conocidos, indican la calidad de atención de un hospital y no la funcionalidad de un sistema de salud. Las limitaciones del trabajo incluyen un sesgo inherente de selección: el centro de estudio es un hospital nacional de referencia. Es posible que pacientes más graves y con patología compleja hayan sido trasladados al centro del estudio. La información sobre el retraso prehospitalario se obtuvo mediante interrogatorio dirigido al paciente y/o familiar y al médico de referencia en el momento del ingreso y podría estar sometida a sesgo de recuerdo.

Este trabajo representa solo una instantánea de un único centro y su entorno geográfico, y sería necesaria una evaluación a nivel poblacional para comprender una situación compleja como las dinámicas del manejo del infarto cardíaco. Sin embargo, este estudio enfatiza la importancia de evaluar este retraso en los sistemas de atención del infarto en otras regiones geográficas y de establecer medidas para disminuir el tiempo de retraso del sistema médico en la atención del infarto agudo de miocardio en México.

\section{Financiamiento}

No se contó con financiamiento para la realización de este trabajo

\section{Conflicto de intereses}

Los autores reportan que no existe conflicto de interés para la elaboración de este trabajo.

\section{Responsabilidades éticas}

Protección de personas y animales. Los autores declaran que para esta investigación no se han realizado experimentos en seres humanos ni en animales.

Confidencialidad de los datos. Los autores declaran que han seguido los protocolos de su centro de trabajo sobre la publicación de datos de pacientes.

Derecho a la privacidad y consentimiento informado. Los autores declaran que en este artículo no aparecen datos de pacientes.

\section{Bibliografía}

1. Martinez-Sánchez C, Borrayo G, Carrillo J, Juarez U, Quintanilla J, Jerjes-Sánchez C. Clinical management and hospital outcomes of acute coronary syndrome patients in Mexico: The Third National Registry of Acute Coronary Syndromes (RENASICA III). Arch Cardiol Mex. 2016; 36:221-324.

2. Martínez-Sánchez C, Arias-Mendoza A, González-Pacheco H, AraizaGaraygordobil D, Marroquín-Donday LA, Padilla-lbarra J, et al. Reperfusion therapy of myocardial infarction in Mexico: A challenge for modern cardiology. Arch Cardiol Mex. 2017;87:144-50.

3. Song JX, Zhu L, Lee CY, Ren H, Cao CF, Chen H. Total ischemic time and outcomes for patients with ST-elevation myocardial infarction: does time of admission make a difference? J Geriatr Cardiol. 2016;13:658-64.

4. Denktas AE, Anderson HV, McCarthy J, Smalling RW. Total ischemic time: the correct focus of attention for optimal ST-segment elevation myocardial infarction care. JACC Cardiovasc Interv. 2011;4:599-604.

5. Dong S, Chu Y, Zhang H, Wang Y, Yang X, Yang L. et al. Reperfusion times of ST-Segment elevation myocardial infarction in hospitals. Pak J Med Sci. 2014;30:1367-71.

6. Alexander T, Mullasari AS, Joseph G, Kannan K, Veerasekar G, Victor SM, et al. A System of care for patients with ST-segment elevation myocardial infarction in India: The Tamil Nadu-ST-Segment Elevation Myocardial Infarction Program. JAMA Cardiol. 2017;2:498-505.

7. Poloński L, Gasior M, Gierlotka M, Kalarus Z, Cieśliński A, Dubiel JS, et al. Polish Registry of Acute Coronary Syndromes (PL-ACS). Characteristics, treatments and outcomes of patients with acute coronary syndromes in Poland. Kardiol Pol. 2007;65:861-72.

8. Danchin N, Coste P, Ferrières J, Steg PG, Cottin Y, Blanchard D, et al; FAST-MI Investigators. Comparison of thrombolysis followed by broad use of percutaneous coronary intervention with primary percutaneous coronary intervention for ST-segment-elevation acute myocardial infarction: data from the french registry on acute ST-elevation myocardial infarction (FAST-MI). Circulation. 2008;118:268-76.

9. Bazzino O, Monaco R, Mario B, Sergio C, Valeria CM, Sergio E, et al. ACCESS Investigators. Management of acute coronary syndromes in developing countries: acute coronary events-a multinational survey of current management strategies. Am Heart J. 2011;162:852-9.

10. Xavier D, Pais P, Devereaux PJ, Xie C, Prabhakaran D, Reddy KS, et al; CREATE registry investigators. Treatment and outcomes of acute coronary syndromes in India (CREATE): a prospective analysis of registry data. Lancet. 2008;371:1435-42. 